\title{
MicroRNA-2 I6a promotes the metastasis and epithelial-mesenchymal transition of ovarian cancer by suppressing the PTEN/AKT pathway
}

This article was published in the following Dove Press journal:

OncoTargets and Therapy

23 May 2017

Number of times this article has been viewed

\author{
Haizhi Liu \\ Ying Pan \\ Xiaoxue Han \\ Jia Liu \\ Ruiman Li \\ Department of Obstetrics and \\ Gynecology, The First Affiliated \\ Hospital of JiNan University, \\ Guangzhou, People's Republic \\ of China
}

Correspondence: Ruiman Li Department of Obstetrics and Gynecology, The First Affiliated Hospital of JiNan University, No 613 Huangpu Road, Guangzhou, People's Republic of China

Email manruili@I63.com
Abstract: MicroRNAs, a group of posttranscriptional regulators of numerous genes, are active participators during the development and progression of ovarian cancer (OC). This study confirmed for the first time that miR-216a was gradually increased in normal, benign, borderline, and OC tissues and that its expression was significantly upregulated in all OC cell lines. Analysis of its clinical association demonstrated that elevated expression of miR-216a was associated with lymph node metastasis and advanced FIGO stage and was correlated with the poor survival of OC patients. Functional experiments showed that miR-216a overexpression potentiated the migration and invasion of CAOV3 cells while miR-216a inhibition reduced the migration and invasion of SKOV-3 cells. Both gain and lose of function assay showed that miR-216a promoted epithelial-mesenchymal transition (EMT) of OC cells. Mechanistically, phosphatase and tensin homolog (PTEN) was confirmed as a direct downstream target of miR-216a in OC cells. Alerting miR-216a expression in OC cells modulated the activity of PTEN/AKT pathway in OC cells. Furthermore, this study confirmed that miR-216a exerted its promoting effects on the metastatic behaviors and EMT of OC cells by inhibiting PTEN/AKT pathway. Taken together, this study demonstrates that miR-216a exerts a promoting role in the metastasis of OC and can serve as a promising biomarker and an attractive therapeutic target of OC.

Keywords: miR-216a, ovarian cancer, PTEN, epithelial-mesenchymal transition, metastasis, biomarker

\section{Introduction}

Ovarian cancer (OC), accounting for the highest cancer-related death in women with gynecologic malignant disease, ${ }^{1}$ is a lethal gynecologic malignancy worldwide. ${ }^{2,3}$ Due to lack of clinical symptoms and diagnostic biomarkers, ${ }^{4}$ most OC patients are already at advanced stages of the disease at the time of diagnosis. ${ }^{5}$ This leads to the poor prognosis and unsatisfactory survival of OC patients, with the mean 5-year survival rate of $\sim 40 \%{ }^{2}$ Therefore, it is critical to identify novel biomarkers and elucidate the molecular mechanisms of the initiation and progression of OC.

MicroRNAs (miRNAs), a group of small noncoding RNAs with 20-22 nucleotides in length, ${ }^{6}$ can regulate the expression of target genes posttranscriptionally and play fundamental roles in regulating tissue-specific protein expression. ${ }^{7}$ Accumulating studies have confirmed that miRNAs play important roles in the development and progression of various human cancers, ${ }^{8-13}$ including OS, and miRNAs have been regarded as promising biomarkers and therapeutic targets of OC. ${ }^{9,14}$

MiR-216a, a novel cancer-related miRNA, has been found to be downregulated in non-small cell lung cancer, ${ }^{15}$ pancreatic cancer, ${ }^{16}$ and oral squamous cell carcinoma, ${ }^{17}$ 
suggesting a tumor suppressor role of miR-216a. However, the expression level and biological functions of miR-216a in $\mathrm{OC}$ and underlying mechanisms for its functional significance remain to be investigated.

In this study, it was found that miR-216a was significantly increased in OC tissues and cells and that elevated miR-216a expression promoted the growth, migration, and invasion of OC cells. It was also confirmed that miR-216a directly downregulated phosphatase and tensin homolog (PTEN) expression by interacting with the PTEN $3^{\prime}$-untranslated region ( 3 '-UTR). Furthermore, it was shown that suppression of PTEN, followed by the activation of AKT pathway, mediated the biological influence of miR-216a in OC.

\section{Methods}

\section{Clinical samples}

A total of 6 normal, 10 benign, 9 borderline, and 87 OC tissue samples were obtained from the Department of Obstetrics and Gynecology, The First Affiliated Hospital of JiNan University. All the patients between January 2006 and December 2009 were enrolled into this study. Clinical samples were used for this study after obtaining informed consent from all the patients. This study was approved by the Institutional Research Ethics Committee of The First Affiliated Hospital of JiNan University.

\section{Cell culture and transfection}

Six human OC cell lines including SKOV-3, HO-8910, A2780, ES-2, CAOV3, and OVCAR3 and an immortalized human fallopian tube epithelial cell line FTE187 cells were obtained from American Type Culture Collection (ATCC, Manassas, VA, USA). OC cell lines were cultured in Dulbecco's Modified Eagle's Medium (DMEM; Gibco Co., New York, USA) supplemented with $10 \%$ fetal bovine serum (FBS) (Gibco Co.), $100 \mathrm{mg} / \mathrm{mL}$ penicillin, and $100 \mathrm{mg} / \mathrm{mL}$ streptomycin. FTE187 cells were cultured in Medium 199 and MCDB105 medium (Sigma-Aldrich Co., St Louis, MO, USA) at the ratio of $1: 1$ containing $10 \% \mathrm{FBS}$ and $10 \mathrm{ng} / \mathrm{mL}$ EGF (Sigma-Aldrich Co.). All cells were kept at $37^{\circ} \mathrm{C}$ humidified atmosphere with $5 \% \mathrm{CO}_{2}$.

The mimic for miR-216a, the inhibitor for miR-216a, and the corresponding control and negative control (NC) vectors were obtained from Genecopoeia (Guangzhou, People's Republic of China). The small interfering RNA (siRNA) specific for PTEN and PTEN expressing vector were obtained from Addgene (Cambridge, MA, USA). All the aforementioned vectors including miR-216a mimics and inhibitors and the PTEN siRNA were transfected into OC cells using
Lipofectamine 2000 based on the manufacturer's instructions (Thermo Fisher Scientific, Waltham, MA, USA).

\section{Real-time quantitative reverse transcription-polymerase chain reaction (qRT-PCR)}

Total RNA from clinical OC tissues and OC cells were extracted using Trizol (Invitrogen). The PCR amplification and quantification for the miR-216a was performed with the TaqMan miRNA Reverse Transcription Kit (Applied Biosystems, Foster City, CA, USA) and TaqMan Human MiRNA Assay Kit (Applied Biosystems). The primers for miR-216a, PTEN, glyceraldehyde-phosphate dehydrogenase (GAPDH), and U6 were purchased from Genecopoeia. U6 was used as the internal control of miR-216a. GAPDH was used as an internal control of PTEN. The relative expression of miR-216a and PTEN was shown as fold difference relative to U6 and GAPDH, respectively.

\section{Western blot}

Whole cell lysates were prepared using radio-immunoprecipitation assay (RIPA) lysis buffer, and protein concentration was measured using the BCA kit (Pierce, Appleton, WI, USA). Generally, $40 \mu \mathrm{g}$ of proteins were loaded, separated, and transferred to PVDF membrane. The following primary antibodies including PTEN $(1: 1,000$; Cell Signaling Technologies, Danvers, MA, USA), AKT $(1: 1,000$; Santa Cruz, CA, USA), p-AKT (1:1,000; Cell Signaling Technologies), and GAPDH (1:1,500, Santa Cruz) were incubated with the membrane overnight at $4^{\circ} \mathrm{C}$. Then, the membranes were incubated with anti-mouse or anti-rabbit secondary antibodies (1:3,000; Santa Cruz). The proteins were visualized using enhanced chemiluminescent (ECL) reagents (Amersham Biosciences Corp., Saint Paul, MN, USA) and the signal was detected using the Bio-Rad Gel imaging system.

\section{Transwell assays}

The migration and invasion of $\mathrm{OC}$ cells were evaluated with Transwell assays. OC cells resuspended in serum-free DMEM were seeded into the upper chamber of the Transwell inserts of $8 \mu \mathrm{m}$ (EMD Millipore, Billerica, MA, USA) with the serum-containing DMEM (20\% FBS) in the lower chamber. For the invasion experiments, the upper chamber was coated with $70 \mu \mathrm{L}$ of DMEM and Matrigel mixture. After $24 \mathrm{~h}$, cells on the upper surface of the chamber were swabbed off while cells on the lower surface were fixed with formaldehyde and stained with crystal violet. The cell 
numbers for the migrated and invaded cells were counted under microscope.

\section{Luciferase reporter assay}

On the day before transfection, $2 \times 10^{5} /$ well OC cells were seeded in 24-well plates. Cells were cotransfected with wild-type PTEN-3'-UTR vector or mutant PTEN-3'-UTR vector along with miR-216a mimic or miR-216a inhibitor, and pRL-SV40 renilla plasmid (Promega Corporation, Fitchburg, WI, USA) using Lipofectamine 2000. After 48 h, the Dual-Luciferase Reporter Assay system (Promega, Shanghai, People's Republic of China) with the luminometer (Promega) was used to measure the activities of firefly and renilla luciferase. Each treatment was performed in triplicate in three independent experiments.

\section{Statistical analysis}

All data were expressed as mean \pm standard error of the mean. Statistical analyses were performed using GraphPad Prism 5.0 (GraphPad Software, Inc., La Jolla, CA, USA). The difference with $P<0.05$ was considered to be statistically significant.

\section{Results}

\section{MiR-2 I 6a was upregulated in OC tissues and cell lines}

The expression levels of miR-216a in 6 normal, 10 benign, 9 borderline, and 87 OC tissue samples were examined. The results of qRT-PCR showed that expression level of miR-216a was gradually increased across the normal, benign, borderline, and OC tissue samples $(P<0.05$, Figure 1A). Then, the expression levels of miR-216a in OC cell lines and the immortalized human fallopian tube epithelial cell line
FTE187 cells were examined. Compared with the FTE187 cells, the expression level of miR-216a in OC cell lines was significantly increased $(P<0.05$, Figure 1B). Among OC cell lines, the expression level of miR-216a was highest in SKOV-3 cells and lowest in CAOV3 cells. These indicate that miR-216a plays an oncogenic role in the development and progression of OC.

\section{Increased miR-216a level is associated with the adverse clinicopathological features and poor prognosis of $\mathrm{OC}$ patients}

After confirming the elevated expression of miR-216a in OC tissues, the clinical significance and prognostic value of miR-216a expression level in OC were examined. As shown in Table 1, high expression level of miR-216a was correlated with the lymph node metastasis $(P<0.01)$ and Federation Internationale of Gynecologie and Obstetrigue (FIGO) stage $(P=0.014)$. Moreover, the Kaplan-Meier analysis showed that patients with high level of miR-216a had significantly decreased overall survival $(P=0.0091$, Figure $2 \mathrm{~A})$ and disease-free survival ( $P=0.0099$, Figure $2 \mathrm{~B})$. These data indicate that elevated miR-216a level is closely associated with the adverse clinical features of OC patients and can potentially serve as effective biomarker for the prognosis of OC patients.

\section{MiR-2 I 6a enhanced the migration and invasion of $O C$ cells}

After confirming the oncogenic role of miR-216a in OC, the functional effects of miR-216a on OC cells were explored. MiR-216a mimic and inhibitor were used to alter the expression level of miR-216a in CAOV3 cells and SKOV-3 cells,
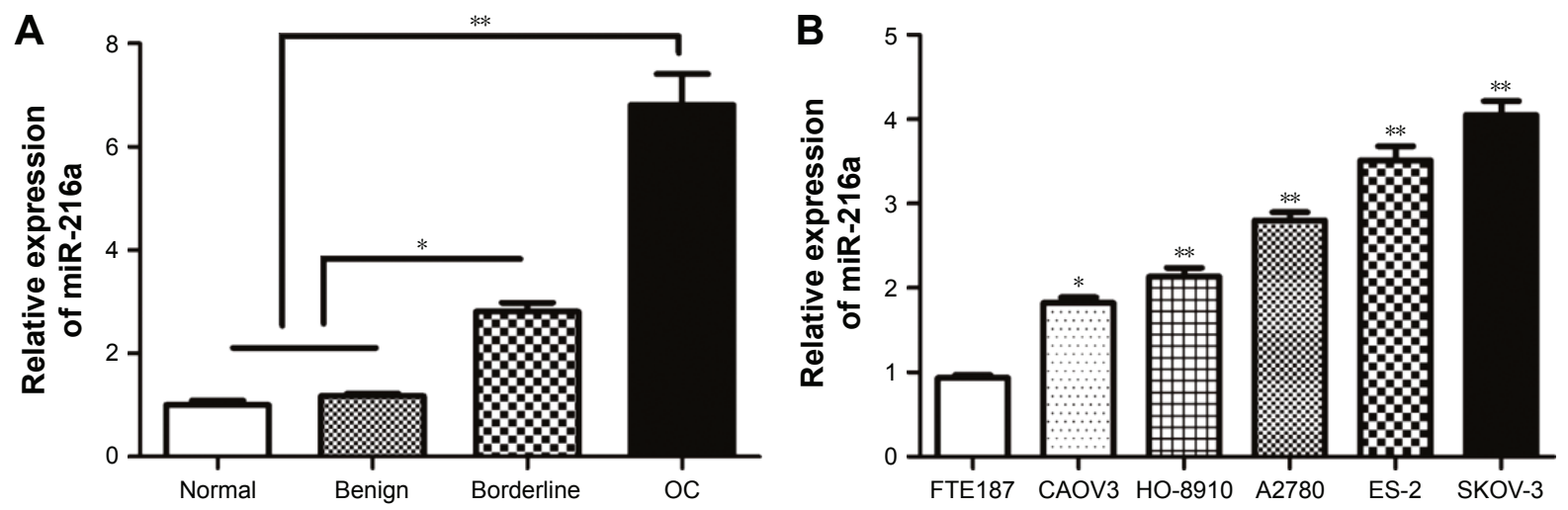

Figure I The expression levels of miR-2I6a in OC tissues and cells.

Notes: (A) The expression of miR-2I6a was compared between normal, benign, borderline, and OC tissues. (B) The expression of miR-2I6a was compared between 5 different OC cell lines (CAOV3, HO-8910, A2780, ES-2, and SKOV-3) and immortalized human fallopian tube epithelial cell line FTEI87 cells. $* P<0.05$, $* * P<0.01$. Abbreviation: OC, ovarian cancer. 
Table I The clinical features of all included patients and the correlations between clinical features and miR-216a expression

\begin{tabular}{|c|c|c|c|c|}
\hline \multirow[t]{2}{*}{ Characteristics } & \multirow{2}{*}{$\begin{array}{l}\text { Number } \\
\text { of patients } \\
(n=87)\end{array}$} & \multicolumn{2}{|c|}{ miR-520g expression } & \multirow[t]{2}{*}{$P$-value } \\
\hline & & $\begin{array}{l}\text { Low group } \\
(n=43)\end{array}$ & $\begin{array}{l}\text { High group } \\
(n=44)\end{array}$ & \\
\hline \multicolumn{4}{|l|}{ Age } & \multirow{3}{*}{0.500} \\
\hline$\leq 50$ & 29 & 16 & 13 & \\
\hline$>50$ & 58 & 27 & 31 & \\
\hline \multicolumn{4}{|l|}{ Ascites } & \multirow[t]{3}{*}{0.644} \\
\hline$\leq 100$ & 27 & 12 & 15 & \\
\hline$>100$ & 60 & 31 & 29 & \\
\hline \multicolumn{4}{|l|}{ Serum CA- 125} & \multirow[t]{3}{*}{0.713} \\
\hline$\leq 35$ & 8 & 3 & 5 & \\
\hline$>35$ & 79 & 40 & 39 & \\
\hline \multicolumn{4}{|l|}{ Tumor size } & \multirow[t]{3}{*}{0.101} \\
\hline$\leq 3 \mathrm{~cm}$ & 61 & 34 & 27 & \\
\hline$>3 \mathrm{~cm}$ & 26 & 9 & 17 & \\
\hline \multicolumn{4}{|c|}{ Lymph node metastasis } & \multirow[t]{3}{*}{$<0.01$} \\
\hline Negative & 49 & 34 & 15 & \\
\hline Positive & 38 & 9 & 29 & \\
\hline \multicolumn{4}{|l|}{ FIGO stage } & \multirow[t]{3}{*}{0.014} \\
\hline I-II & 12 & 10 & 2 & \\
\hline III-IV & 75 & 33 & 42 & \\
\hline
\end{tabular}

Abbreviation: FIGO, Federation Internationale of Gynecologie and Obstetrigue.

respectively. Then the change of migration and invasion of OC cells were examined after overexpressing or inhibiting miR-216a. Transfection of miR-216a mimic significantly increased the expression level of miR-216a in CAOV3 cells $(P<0.01$, Figure 3A). Functionally, the migration and invasion of OC cells were also significantly increased after transfection of miR-216a mimic into CAOV 3 cells $(P<0.05$, Figure 3B). On the other hand, the expression of miR-216a was significantly inhibited in SKOV-3 cells after transfection of miR-216a inhibitor $(P<0.01$, Figure $3 C)$. Subsequently,

A

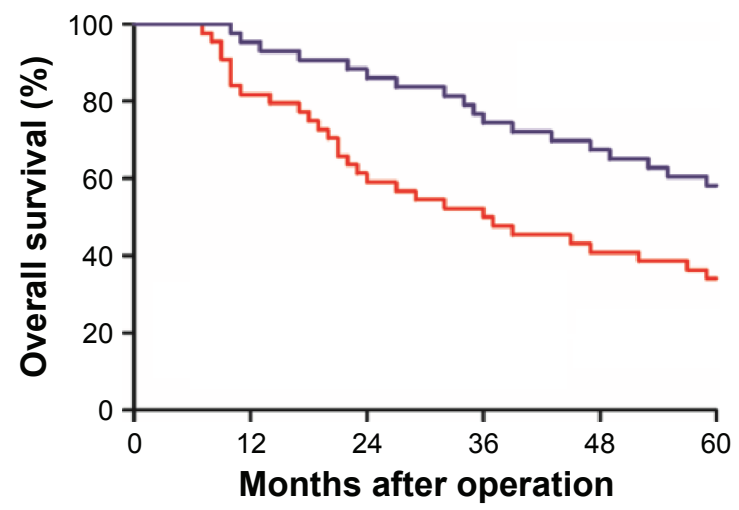

the migration $(P<0.05$, Figure 3D) and invasion $(P<0.05$, Figure 3D) of SKOV-3 cells were significantly decreased.

\section{MiR-2I6a promotes the epithelial- mesenchymal transition (EMT) of OC cells}

EMT has been a well-recognized mechanism for the metastasis of cancer cells. An emerging evidence has confirmed that miRNAs are critical regulators of the EMT process of cancer cells. Therefore, this study examined whether miR-216a could promote the metastasis of OC cells by modulating EMT. Overexpression of miR-216a in CAOV3 cells led to significantly decreased expression of E-cadherin $(P<0.01$, Figure $4 \mathrm{~A})$ and increased level of Vimentin $(P<0.01$, Figure 4A). On the other hand, inhibition of miR-216a resulted in significantly elevated level of E-cadherin $(P<0.01$, Figure 4B) and reduced level of Vimentin $(P<0.05$, Figure 4B). These data indicate that miR-216a can promote the metastasis of OC by regulating EMT process.

\section{PTEN is a direct downstream target of miR-2I6a in OC cells}

To further elucidate the molecular mechanisms underlying the promoting effects of miR-216a on the metastasis of OC cells, two publicly available databases (TargetScan 6.2 and miRanda) were searched to identify the downstream target of miR-216a, and it was found that PTEN was predicted to be the downstream target of miR-216a since the 3'-UTR of PTEN mRNA contained the complementary sequence of miR-216a (Figure 5A). To confirm that miR-216a could directly target the 3'-UTR of PTEN, luciferase reporter assay was performed

B

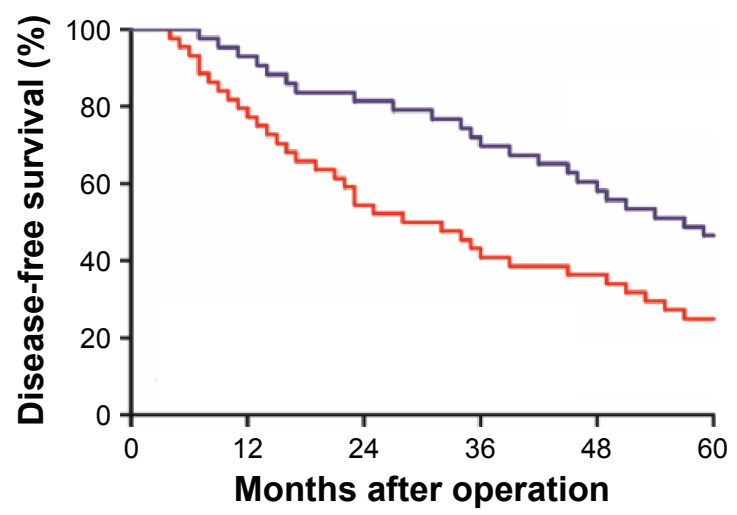

- MiR-216a low group — MiR-216a high group

Figure 2 The prognostic value of miR-2I6a for OC patients.

Note: (A) Overall survival and (B) disease-free survival were compared between patients of high level of miR-2I6a and those of low level of miR-2I6a. Abbreviation: OC, ovarian cancer. 

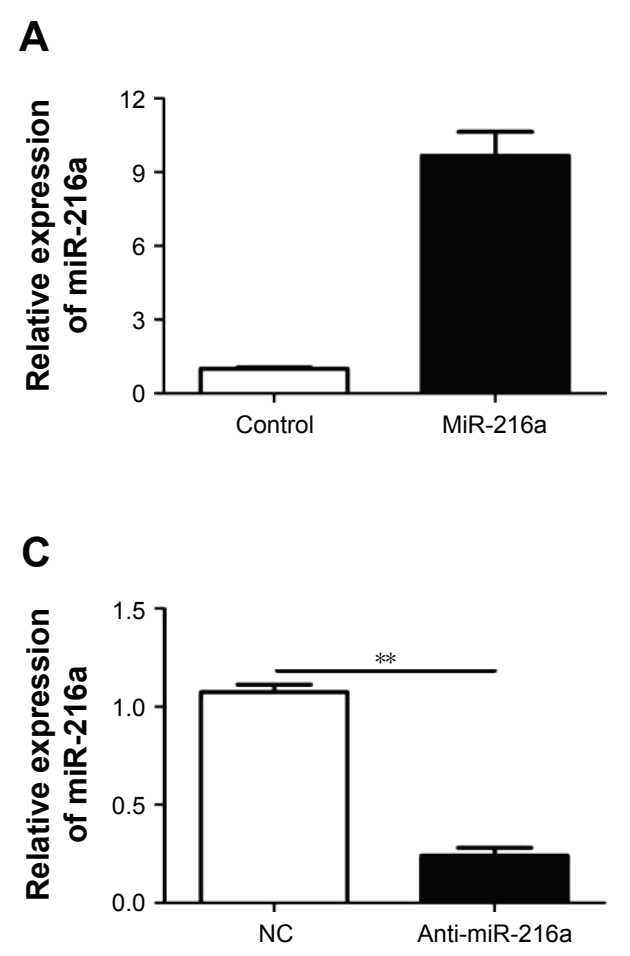
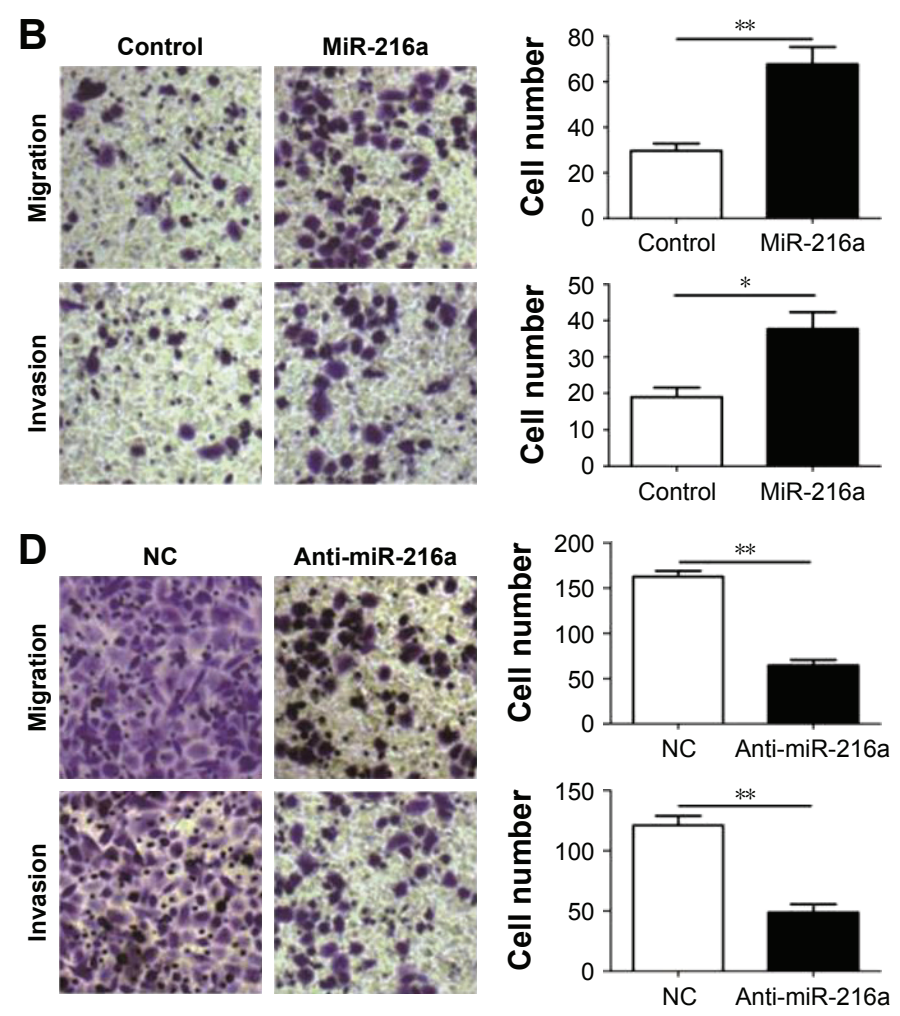

Figure 3 MiR-216a promotes the migration and invasion of OC cells.

Notes: (A) MiR-2I6a mimics significantly elevated the expression level of miR-2I6a in CAOV3 cells. (B) Overexpression of miR-2I6a significantly increased the migration and invasion of CAOV3 cells. $* P<0.05, * * P<0.01$. (C) MiR-2I 6a inhibitor significantly decreased the expression level of miR-216a in SKOV-3 cells. $* * P<0.01$. (D) Downregulation of miR-2I6a significantly reduced the migration and invasion of SKOV-3 cells. $* * P<0.01$.

Abbreviations: OC, ovarian cancer; NC, negative control.
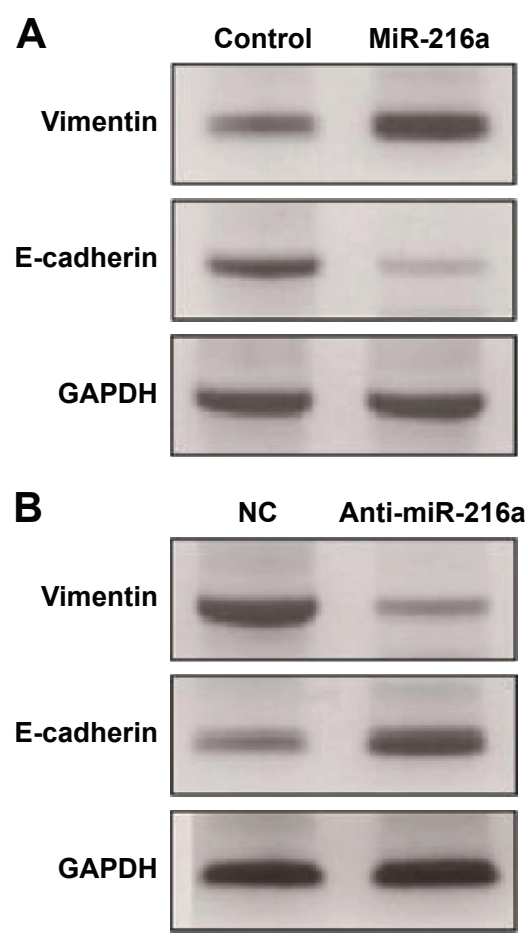

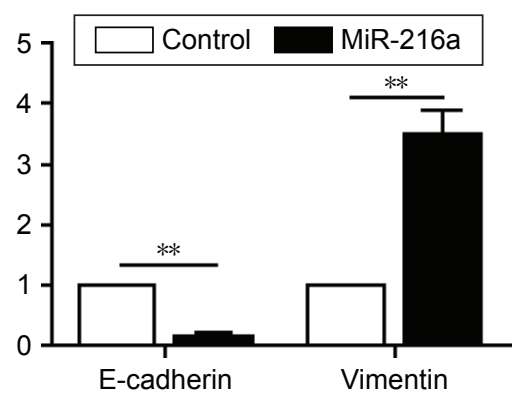

E-cadherin Vimentin

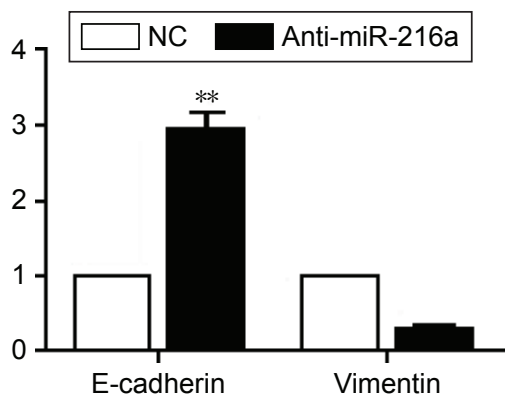

Figure 4 MiR-2I6a promotes the EMT phenotype of OC cells.

Notes: (A) Western blot results of E-cadherin and Vimentin in CAOV3 cells transfected with miR-216a mimics; $* * P<0.01$. (B) Western blot results of E-cadherin and Vimentin in SKOV-3 cells transfected with miR-2I6a inhibitor; ${ }^{* * P}<0.01$.

Abbreviations: EMT, epithelial-mesenchymal transition; OC, ovarian cancer; NC, negative control. 


\section{A 3' UGUCAACGGUCGACUCUAAU 5' \\ | | | || \\ 5' AAAUUUUCAAUUUGAGAUUc 3' \\ 5' AAAUUUUCAAUUU $\underline{A} A \underline{C} A \underline{A} U \mathrm{U} c 3^{\prime}$}

B

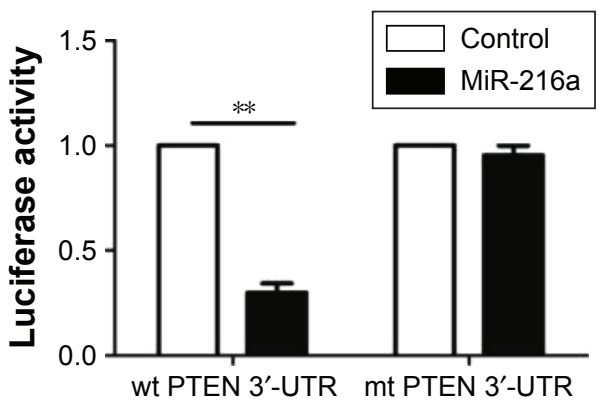

D
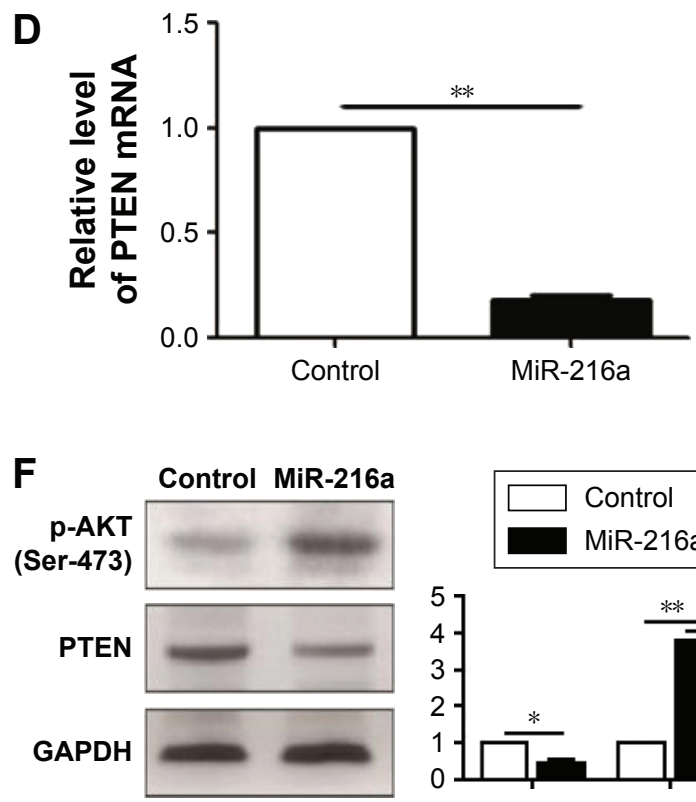

C

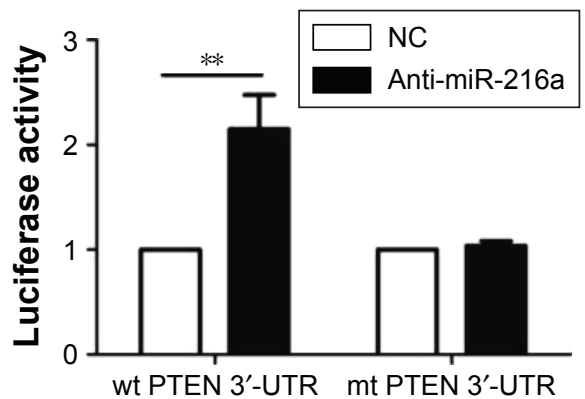

E
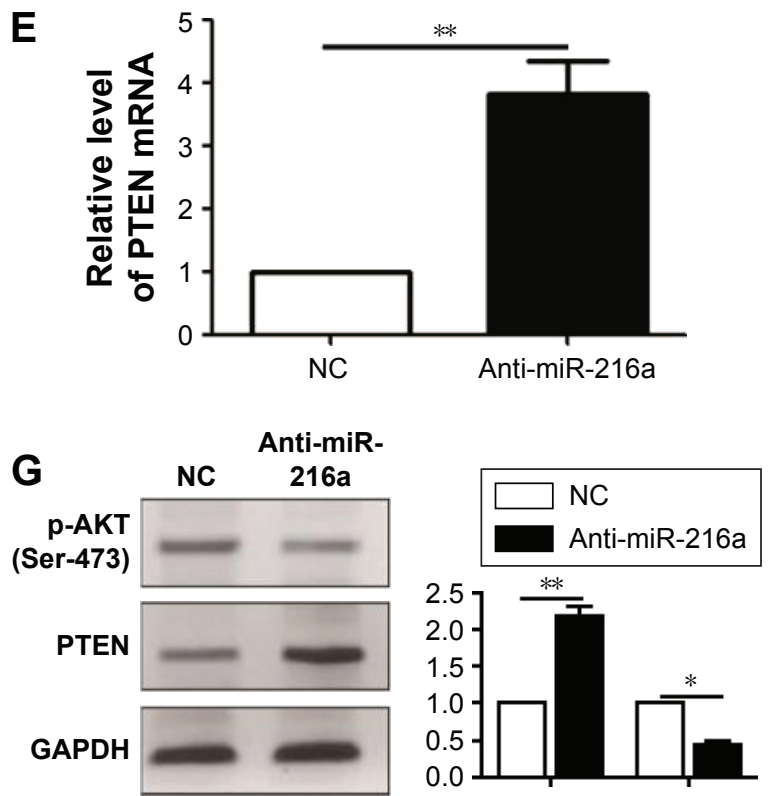

Figure 5 PTEN is a direct target of miR-216a in OC cells.

Notes: (A) MiR-2I6a and its complementary sequence in the $3^{\prime}$-UTR of PTEN. (B) Overexpression of miR-2I6a significantly inhibited the luciferase activity that carried wt $3^{\prime}$-UTR of PTEN but had no obvious influence on $\mathrm{mt} 3^{\prime}$-UTR of PTEN in CAOV3 cells. $* * P<0.01$. (C) Inhibition of miR-2I6a obviously increased the luciferase activity that carried wt 3'-UTR of PTEN but had no obvious influence on mt 3'-UTR of PTEN in SKOV-3 cells. **P $<0.01$. (D) Overexpression of miR-2I6a significantly decreased PTEN mRNA level in CAOV3 cells. ${ }^{* * P}<0.0 \mathrm{I}$. (E) Inhibition of miR-2I6a significantly increased PTEN mRNA level in SKOV-3 cells. $* * P<0.01$. (F) Overexpression of miR-2I6a significantly inhibited the expression of PTEN and increased $P$-AKT in CAOV3 cells. $* P<0.05, * * P<0.01$. (G) Inhibition of miR-2I6a significantly increased the expression of PTEN and decreased $\mathrm{p}-\mathrm{AKT}$ in SKOV-3 cells. $* \mathrm{P}<0.05,{ }^{*} * \mathrm{P}<0.01$.

Abbreviations: OC, ovarian cancer; PTEN, phosphatase and tensin homolog; wt, wild type; mRNA, messenger RNA; NC, negative control; mt, mutant.

in OC cells. The results of luciferase assay showed that forced expression of miR-216a in CAOV3 cells significantly decreased the luciferase activity of wild-type $3^{\prime}$-UTR of PTEN $(P<0.01$, Figure 5B) without having obvious effects on that of mutant 3'-UTR of PTEN (Figure 5B). On the other hand, inhibition of miR-216a in SKOV-3 cells obviously increased the luciferase activity of the wild-type $3^{\prime}$-UTR of PTEN $(P<0.05$, Figure 5C) while it had no significant influence on that of mutant 3'-UTR of PTEN (Figure 5C). Furthermore, the study examined whether miR-216a could modulate the expression of PTEN in OC cells. qRT-PCR showed that
miR-216a overexpression significantly decreased the level $(P<0.05$, Figure 5D) of PTEN mRNA in CAOV3 cells while miR-216a knockdown remarkably increased the mRNA level $(P<0.05$, Figure 5E) of PTEN in SKOV-3 cells. The results of western blot (WB) showed that overexpression of miR-216a significantly decreased the expression $(P<0.05$, Figure 5F) of PTEN in CAOV3 cells while inhibition of miR-216a remarkably increased the protein level $(P<0.05$, Figure 5G) of PTEN in SKOV-3 cells. Additionally, the phosphorylation (Ser-473) of Akt, which was under the regulation of PTEN, was increased after the overexpression of miR-216a 
$(P<0.05$, Figure 5F) and decreased after the inhibition of miR-216a $(P<0.05$, Figure 5G). Taken together, these data demonstrate that PTEN is a direct downstream target of miR$216 \mathrm{a}$ in OC cells.

\section{MiR-2I6a exerts its oncogenic role in OC by inhibiting PTEN/AKT pathway}

After confirming PTEN was a direct target of miR-216a in OC cells, this study further investigated whether PTEN could mediate the functional effects of miR-216a on OC cells. PTEN expressing vector was transfected into CAOV3 cells overexpressing miR-216a (CAVO-3-miR-216a cells). WB results showed that PTEN vector significantly increased PTEN expression level in CAOV3-miR-216a cells along with significant decrease of p-AKT $(P<0.05$, Figure 6A). Subsequently, overexpression of PTEN partly abrogated the promoting effects of miR-216a on EMT of CAOV 3 cells, as suggested by increased expression of E-cadherin $(P<0.05$, Figure 6A) and decreased level of Vimentin $(P<0.05$, Figure 6A). Transwell assay showed that overexpression of PTEN partly abrogated the promoting effects of miR-216a on the migration and invasion of CAOV 3 cells $(P<0.05$, respectively, Figure 6B). On the other aspect, PTEN-specific siRNA was transfected into SKOV-3 cells expressing miR-216a inhibitor (SKOV-3-anti-miR-216a cells). As suggested by WB, PTEN siRNA led to significant suppression of PTEN expression $(P<0.05$, Figure 6C) and increased level of p-AKT $(P<0.05$, Figure 6C) in SKOV-3-anti-miR-216a cells. Furthermore, inhibition of PTEN along with increased p-AKT partly reversed the suppressive effects of miR-216a inhibition on EMT $(P<0.05$, Figure 6C), migration, and invasion of SKOV-3-anti-miR-216a cells $(P<0.05$, respectively, Figure 6D). Taken together, these indicate that the prompting effects of miR-216a on the metastatic behaviors and EMT of OC cells were mediated by inhibiting PTEN/ AKT pathway.

\section{Discussion}

Increasing evidence has demonstrated that aberrant miRNA expression was directly associated with the development and progression of human cancers by acting as either oncogenes or tumor suppressors. ${ }^{18,19}$ Moreover, miRNAs have been recognized as attractive biomarkers and therapeutic targets of human cancers including OC. ${ }^{20} \mathrm{MiR}-216 \mathrm{a}$ has been found to be an active participator in human malignancies. ${ }^{15-17,21}$ It was found to inhibit the growth and metastasis of oral squamous cell carcinoma by inhibiting eukaryotic translation initiation factor $4 \mathrm{~B} .{ }^{17}$ In pancreatic cancer, it was found to inhibit the growth of pancreatic cancer by inhibiting JAK2. ${ }^{16}$ However, in liver cancer, miR-216a was found to induce EMT and regulate the drug resistance, suggesting an oncogenic role of miR-216a. ${ }^{21}$ This study confirmed that miR-216a was significantly elevated in OC tissues and cell lines and found that elevated level of miR-216a was correlated with adverse clinical features including lymph node metastasis and advanced FIGO stage. Furthermore, survival analysis showed that high level of miR-216a was closely associated with the poor survival of OC patients. Therefore, miR-216a plays an oncogenic role and can potentially serve as promising biomarker and prognostic indicator in OC.

Functionally, miR-216a was found to be involved in the growth of pancreatic cancer and oral squamous cell carcinoma. ${ }^{16,17}$ It was also found to participate in the drug resistance of liver cancer. ${ }^{21}$ This study found that miR-216a could regulate the migration and invasion of OC cells. Furthermore, this study confirmed that miR-216a could promote the EMT of OC cells. These indicate that miR-216a plays an oncogenic role in $\mathrm{OC}$ by potentiating the metastatic behaviors and EMT of OC cells.

PTEN, which is a well-known tumor suppressor, has been found to play an important role in the development and progression of various human cancers. ${ }^{22-24}$ This study demonstrated that miR-216a could directly modulate the expression of PTEN by interacting with its $3^{\prime}$-UTR. First, the complementary sequences of miR-216a could be found in the 3 '-UTR of PTEN. Second, altering miR-216a level could significantly influence the luciferase activity of wildtype 3'-UTR of PTEN while it had no obvious effect on that of mutant (mt) 3'-UTR of PTEN. Third, overexpression of miR-216a resulted in significantly decreased expression level of PTEN while suppression of miR-216a led to obviously increased expression level of PTEN. Additionally, the phosphorylation (Ser-473) of AKT, which was under the regulation of PTEN, was altered accordingly after the overexpression or inhibition of miR-216a. These solidly supported the conclusion that PTEN was a direct downstream target of miR-216a in OC, and miR-216a could modulate the PTEN/AKT pathway in OC cells. Furthermore, it was found that overexpressing PTEN in OC cells expressing miR-216a mimic could abrogate the promoting effects of miR-216a overexpression on the metastasis and EMT of OC cells while PTEN siRNA could reverse the inhibitory effect of miR-216a inhibitor on these metastatic ability of OC cells. Therefore, these data indicate that PTEN was not only the downstream target of miR-216a but also the functional mediator of miR-216a in OC. 


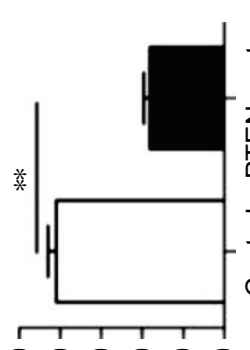

응ㅇㅇㅇㅇㅇ دәqunu ||ә弓
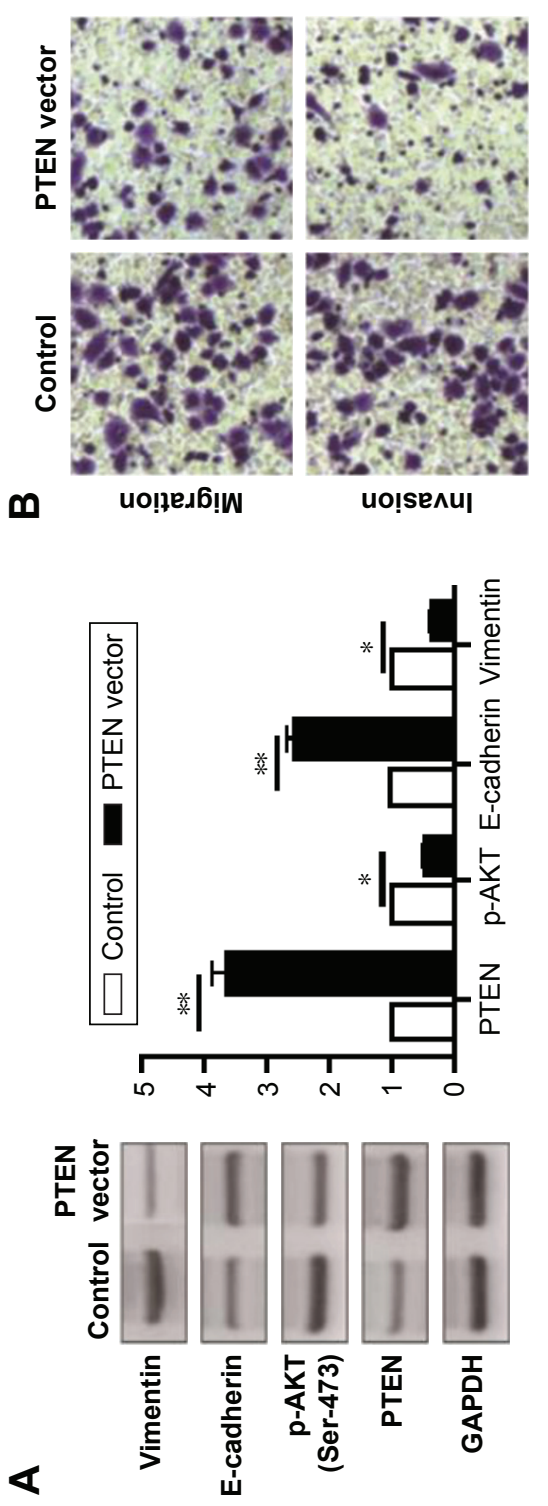
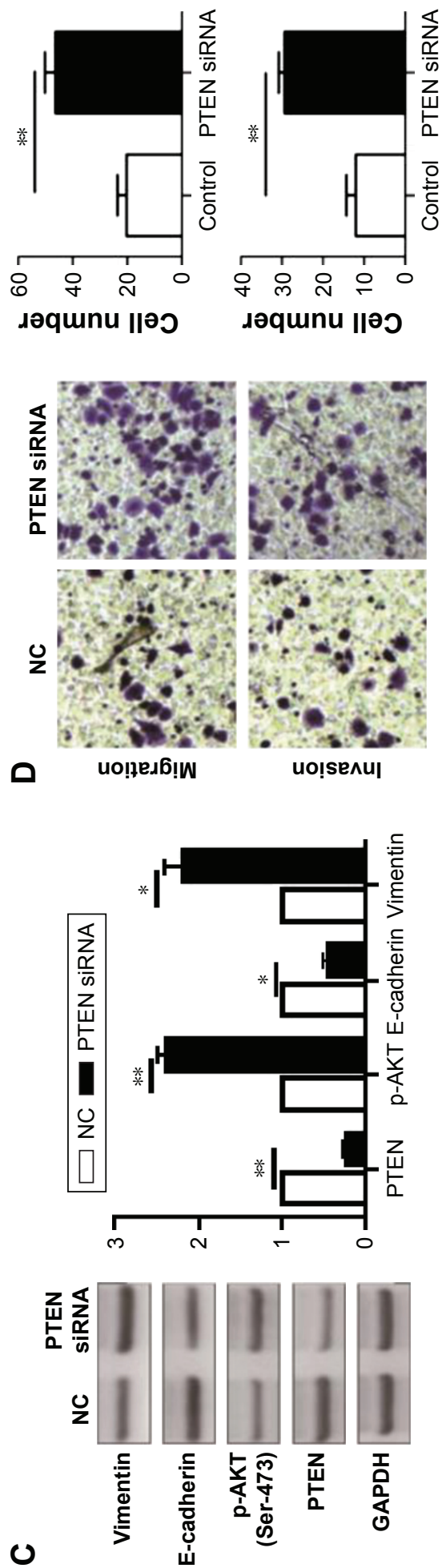

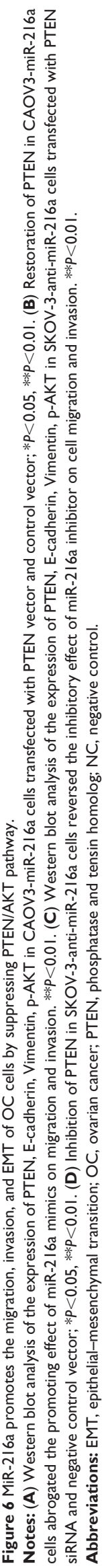




\section{Conclusion}

This study finds that miR-216a is significantly increased in OC tissues and cells. Elevated expression of miR-216a is associated with adverse clinical features and poor prognosis of OC patients. Functionally, miR-216a can promote the migratory and invasive ability and EMT of OC cells. Furthermore, this study confirms that PTEN is a downstream target of miR-216a in OC cells, and miR-216a exerts its promoting effects on the metastasis and EMT of OC cells by regulating PTEN/AKT pathway.

\section{Acknowledgment}

This study was supported by Scientific Research Foundation of Henan (No 092102310090).

\section{Disclosure}

The authors report no conflicts of interest in this work.

\section{References}

1. Hennessy BT, Coleman RL, Markman M. Ovarian cancer. Lancet. 2009;374(9698):1371-1382.

2. DeSantis CE, Lin CC, Mariotto AB, et al. Cancer treatment and survivorship statistics, 2014. CA Cancer J Clin. 2014;64(4):252-271.

3. Bast RC, Hennessy B, Mills GB. The biology of ovarian cancer: new opportunities for translation. Nat Rev Cancer. 2009;9(6):415-428.

4. Mezzanzanica D. Ovarian cancer: a molecularly insidious disease. Chinese J Cancer. 2015;34(1):1.

5. Holschneider $\mathrm{CH}$, Berek JS. Ovarian cancer: epidemiology, biology, and prognostic factors. Paper presented at: Seminars in Surgical Oncology; 2000.

6. Bartel DP. MicroRNAs: genomics, biogenesis, mechanism, and function. Cell. 2004;116(2):281-297.

7. Mendell JT. MicroRNAs: critical regulators of development, cellular physiology and malignancy. Cell Cycle. 2005;4(9):1179-1184.
8. Chong GO, Jeon H-S, Han HS, et al. Differential microRNA expression profiles in primary and recurrent epithelial ovarian cancer. Anticancer Res. 2015;35(5):2611-2617.

9. Llauradó M, Majem B, Altadill T, et al. MicroRNAs as prognostic markers in ovarian cancer. Mol Cell Endocrinol. 2014;390(1):73-84.

10. Lu J, Getz G, Miska EA, et al. MicroRNA expression profiles classify human cancers. Nature. 2005;435(7043):834-838.

11. Zhang $\mathrm{S}, \mathrm{Lu} \mathrm{Z}$, Unruh $\mathrm{AK}$, et al. Clinically relevant microRNAs in ovarian cancer. Mol Cancer Res. 2015;13(3):393-401.

12. Iorio MV, Visone R, Di Leva G, et al. MicroRNA signatures in human ovarian cancer. Cancer Res. 2007;67(18):8699-8707.

13. Calin GA, Croce CM. MicroRNA signatures in human cancers. Nat Rev Cancer. 2006;6(11):857-866.

14. Mezzanzanica D, Bagnoli M, De Cecco L, Valeri B, Canevari S. Role of microRNAs in ovarian cancer pathogenesis and potential clinical implications. Int J Biochem Cell Biol. 2010;42(8):1262-1272.

15. Wang R-T, Xu M, Xu C-X, Song Z-G, Jin H. Decreased expression of miR216a contributes to non-small-cell lung cancer progression. Clin Cancer Res. 2014;20(17):4705-4716.

16. Wang S, Chen X, Tang M. MicroRNA-216a inhibits pancreatic cancer by directly targeting Janus kinase 2. Oncol Rep. 2014;32(6):2824-2830.

17. Li L, Ma HQ. MicroRNA-216a inhibits the growth and metastasis of oral squamous cell carcinoma by targeting eukaryotic translation initiation factor 4B. Mol Med Rep. 2015;12(2):3156-3162.

18. Gregory RI, Shiekhattar R. MicroRNA biogenesis and cancer. Cancer Res. 2005;65(9):3509-3512.

19. Sassen S, Miska EA, Caldas C. MicroRNA - implications for cancer. Virchows Archiv. 2008;452(1):1-10.

20. Corney DC, Nikitin AY. MicroRNA and ovarian cancer. Histol Histopathol. 2008;23(9):1161.

21. Xia H, Ooi LLP, Hui KM. MicroRNA-216a/217-induced epithelialmesenchymal transition targets PTEN and SMAD7 to promote drug resistance and recurrence of liver cancer. Hepatology. 2013;58(2): 629-641.

22. Chalhoub N, Baker SJ. PTEN and the PI3-kinase pathway in cancer. Ann Rev Pathol. 2009;4:127.

23. Di Cristofano A, Pandolfi PP. The multiple roles of PTEN in tumor suppression. Cell. 2000;100(4):387-390.

24. Carnero A, Blanco-Aparicio C, Renner O, Link W, Leal JF. The PTEN/ PI3K/AKT signalling pathway in cancer, therapeutic implications. Curr Cancer Drug Targets. 2008;8(3):187-198.
OncoTargets and Therapy

\section{Publish your work in this journal}

OncoTargets and Therapy is an international, peer-reviewed, open access journal focusing on the pathological basis of all cancers, potential targets for therapy and treatment protocols employed to improve the management of cancer patients. The journal also focuses on the impact of management programs and new therapeutic agents and protocols on

\section{Dovepress}

patient perspectives such as quality of life, adherence and satisfaction. The manuscript management system is completely online and includes a very quick and fair peer-review system, which is all easy to use. Visit http://www.dovepress.com/testimonials.php to read real quotes from published authors. 